To try to reproduce, and thereby capture, this type of unpublished information, Moosavi et al. used a robotic system to run a series of experiments that explores the effects of different reaction conditions - changing the solvent, temperature, reactant concentration and so on - in the synthesis of a widely used, copper-based MOF known as HKUST-1 (ref. 4). The robot could run 30 reactions per day, and thereby obtained a data set of conditions that led to successful and failed reactions. The data were then processed by an algorithm that mimics genetic and evolutionary processes: each iteration of the algorithm applies a selection pressure to the data that causes evolved conditions to emerge as a result of 'survival of the fittest'.

Moosavi and colleagues performed 3 rounds of 30 experiments, using the algorithm and the quality of the MOF samples produced in each round to guide the conditions for the subsequent rounds. The authors thereby identified an optimized procedure for making HKUST-1, yielding material that had superb crystallinity (Fig. 1) and phase purity (which measures the proportions of different crystal forms present in the material), and a high surface area - all of which are desirable properties for applications of MOFs. Ordinarily, only the optimized conditions would be published in the literature, with all the other reactions being lost within the confines of a dusty notebook (or perhaps, these days, buried in the digital archives of an e-notebook).

Instead, Moosavi et al. returned to the suboptimal reactions, and used machine learning to analyse them. In this way, the authors identified the reaction parameters that have the largest effect on the quality of the resulting MOFs. For example, they found that changes in reaction temperature have a much greater effect on the crystallinity and surface area of the products than have the stoichiometry of the linkers and nodes used in the reaction. By ranking and analysing the relative importance of nine reaction metrics, the authors generated information akin to a chemist's intuition.

Moosavi et al. used this chemical intuition to develop a synthesis of Zn-HKUST-1, which is a MOF that has the same structure as HKUST-1, but with zinc nodes instead of copper ones. This might sound like a trivial challenge, especially considering the chemical similarities between copper and zinc. However, the authors found that the top ten most widely used reaction conditions for synthesizing HKUST-1 all failed to produce Zn-HKUST-1. This sort of situation is tremendously frustrating for chemists, who typically must then work out how to obtain the desired material from scratch, by trying out many different sets of reaction conditions.

By contrast, Moosavi et al. focused on the dominant reaction parameters identified by machine learning, and discovered two sets of conditions that produce Zn-HKUST-1 after just 20 trial reactions. The authors suggest that taking a completely unguided approach would have required thousands of reactions to achieve the same result.

Moosavi and colleagues acknowledge that the data produced in their experiments are ideal for machine-learning analysis their robotic set-up precisely controls the reaction parameters, reducing variables in the reaction outcomes, and only one synthetic reaction was considered. Data produced from a more disparate set of synthetic reactions would have been more difficult to handle. Moreover, the authors focused on the quality of the MOF materials, but did not report other key outcomes, such as reaction yields. Lowyielding MOF syntheses would be impractical, even if they produce the most pristine materials known.

Nevertheless, Moosavi and colleagues' work has the potential to greatly improve and accelerate the synthesis of MOFs. The authors have made their software available online (see go.nature.com/2dtppxn), so that chemists can contribute to collective chemical intuition by reporting successful and failed reaction conditions. This kind of group engagement should be applauded, and could greatly benefit the MOF community. Now, if only they could improve my intuition at the roulette table...

Seth Cohen is in the Department of Chemistry and Biochemistry, University of California, San Diego, La Jolla, California 92093-0358, USA.

e-mail:scohen@ucsd.edu

1. Moosavi, S. M. et al. Nature Commun. 10, 539 (2019).

2. Jiang, J., Zhao, Y. \& Yaghi, O. M. J. Am. Chem. Soc. 138, 3255-3265 (2016).

3. Furukawa, H., Cordova, K. E., O'Keeffe, M. \& Yaghi, O. M. Science 341, 1230444 (2013)

4. Chui, S. S.-Y., Lo, S. M.-F., Charmant, J. P. H.

Orpen, A. G. \& Williams, I. D. Science 283,

1148-1150 (1999).

\title{
Multiple sclerosis enters a grey area
}

\begin{abstract}
Studies of multiple sclerosis have long focused on the white matter of the brain. Insights into how immune cells target the brain's grey matter now illuminate the stage of the disease at which neurodegeneration occurs. SEE ARTICLE P.503
\end{abstract}

\section{JENNA L. PAPPALARDO \& DAVID A. HAFLER}

$\mathrm{M}$ ultiple sclerosis is an autoimmune disease with a genetic origin. The condition is characterized by an attack on the brain mediated by the immune system, leading to diverse symptoms caused by damage to neurons ${ }^{1}$. Animal models are available for the early stage of the disease, which is called relapsing-remitting multiple sclerosis. However, the progressive forms of multiple sclerosis associated with neurodegeneration and disability are comparatively understudied, and what shapes the course of disease progression is largely unknown. Insights into the later stage of the condition could help the development of clinical approaches that tackle the underlying causes. On page 503, Lodygin et al. ${ }^{2}$ shed light on the progressive stage of the disease. Using a rat model and blood samples from people who had multiple sclerosis, the authors identify the protein target of an immune cell that attacks the brain region called grey matter.

Multiple sclerosis is usually diagnosed at the relapsing-remitting stage, in which people have periods of symptoms and then remission when the symptoms subside. There is growing evidence that relapsing-remitting multiple sclerosis originates from inflammation that is driven by immune cells ${ }^{3}$. A hallmark of this disease stage is a targeted attack by immune cells, including $\mathrm{T}$ cells and macrophages, on the brain region called white matter, which contains parts of neurons known as axons or nerve fibres (Fig. 1). Myelin, a mixture of lipids and proteins that covers and protects nerve cells in the white matter, is thought to be the T-cell target in this destructive process ${ }^{4}$. Much progress has been made in understanding how this process occurs ${ }^{5-7}$, particularly from studies of a mouse model called experimental autoimmune encephalomyelitis.

Relapsing-remitting multiple sclerosis often leads to the progressive stage of the disease. If this progression occurs, people stop experiencing remission periods and have a gradual loss of nerve-cell function that might be linked to destruction in the brain's grey-matter region ${ }^{8,9}$; grey matter harbours the parts of nerve cells called cell bodies, which contain the nucleus. Immunosuppressive treatment for relapsing-remitting multiple sclerosis can help to decrease the chance of progressive disease developing ${ }^{10}$, but what causes this switch is not understood.

The destruction of material in white and grey matter in people with multiple sclerosis might arise from distinct inflammatory processes ${ }^{8}$. What drives immune-system cells to infiltrate 


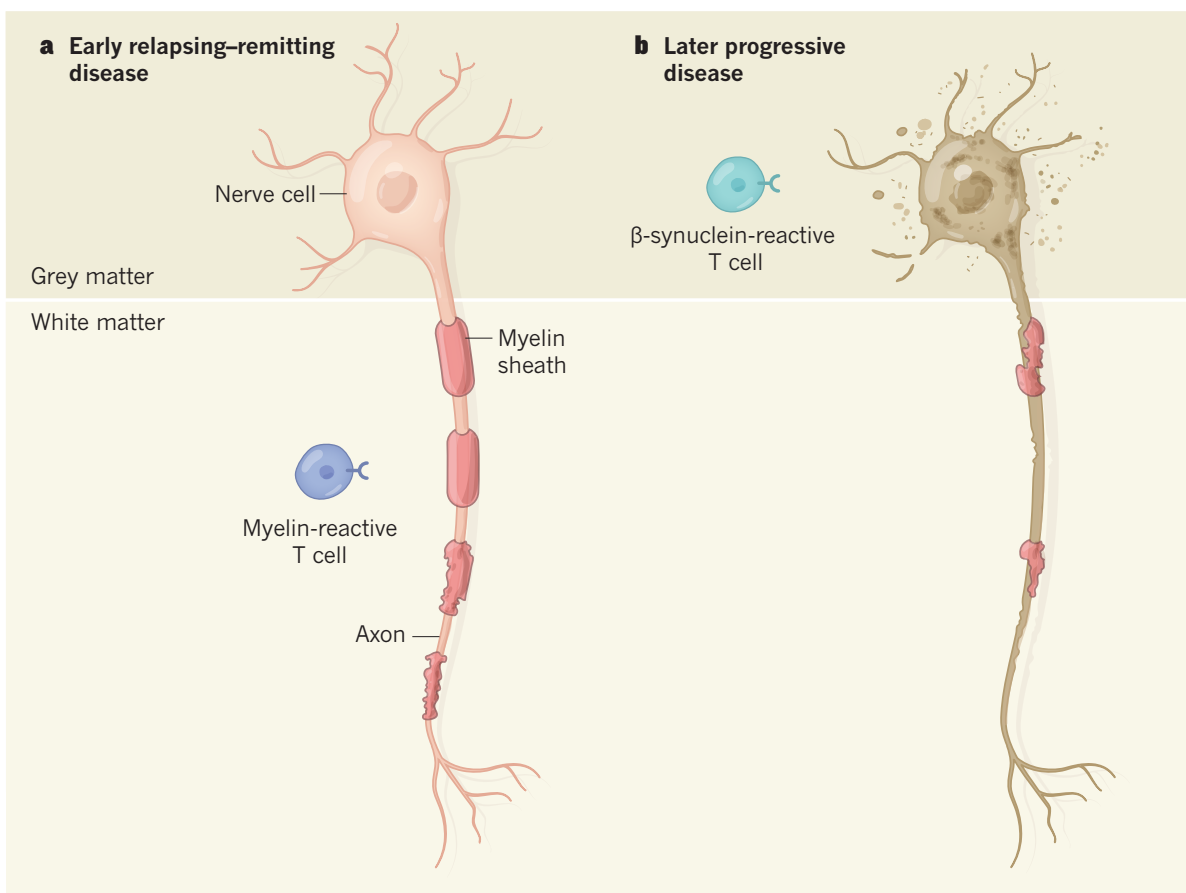

Figure 1 | Immune cells and multiple sclerosis. a, The myelin sheath (a mixture of lipids and proteins) covers and protects the axonal region of nerve cells in the part of the brain called white matter. Immune cells called T cells that recognize myelin have a key role in initiating an autoimmune destructive attack on myelin that is the hallmark of the early-stage disease - relapsing-remitting multiple sclerosis. b, Lodygin et al. ${ }^{2}$ shed light on later-stage, progressive multiple sclerosis, which is associated with disability.

Progressive disease is characterized by destruction of the part of nerve cells that contains the nucleus and is located in the brain's grey-matter region. Studying a rat model of the disease and blood samples from people who had multiple sclerosis, the authors report that progressive disease is characterized by the presence of $\mathrm{T}$ cells that recognize the protein $\beta$-synuclein, which is present in grey matter.

and damage these different brain regions is therefore a key question. Lodygin and colleagues investigated this using a rat model to compare the migration into the brain of $\mathrm{T}$ cells that target myelin and those that recognize the protein $\beta$-synuclein, which is present in grey matter and is a possible target ${ }^{11}$ for autoimmune attack. It was thought that immune cells might be drawn to different brain regions on the basis of the cells' expression of receptors for inflammatory chemokine molecules that can influence immune-cell movement.

The authors reveal that $\mathrm{T}$-cell specificity for its target protein, rather than the expression of chemokine receptors on $\mathrm{T}$ cells, influences the location of these cells in the brain. Myelinreactive $\mathrm{T}$ cells were found in myelin-rich white-matter regions of the rat brain and did not substantially infiltrate or damage the grey matter. By contrast, $\mathrm{T}$ cells that recognize $\beta$-synuclein caused damage mainly in the grey matter, where they released cytokines and induced tissue damage in neurons and neuronsupporting glial cells. The grey-matter damage was permanent, mirroring neurodegenerative processes that occur in multiple sclerosis and other neurodegenerative diseases, such as Parkinson's disease, in which the grey matter is mainly affected.

By identifying $\mathrm{T}$ cells that are linked to damage in grey matter in a model of multiple sclerosis, Lodygin and colleagues' work suggests a new way to study disease progression. This is particularly useful because the understanding of molecules targeted in grey matter has lagged behind knowledge of those targeted in white matter.

The authors assessed the levels of myelinreactive and $\beta$-synuclein-reactive T cells in blood samples from people with multiple sclerosis and healthy controls. People who had relapsing-remitting multiple sclerosis had more myelin-reactive $\mathrm{T}$ cells than did the healthy controls, whereas people who had progressive multiple sclerosis had more $\beta$-synuclein-reactive T cells than did the healthy controls. The number of $\beta$-synucleinreactive $\mathrm{T}$ cells was highest in people who had had the disease for a longer time. This suggests that $\beta$-synuclein might be a late-stage autoimmune target that arises through a process called epitope spreading, in which an initial immune response that targets a particular protein is followed by a diversified immune response that targets other proteins.

Variability is often observed in the presence and degree of white-matter and grey-matter destruction in relapsing-remitting and progressive multiple sclerosis, which might reflect the action of $\mathrm{T}$ cells of differing protein specificities. This issue could be examined by using magnetic resonance imaging to determine whether there is a correlation between the frequency of myelin-reactive compared with $\beta$-synuclein-reactive $\mathrm{T}$ cells and the location and extent of the brain damage observed. Moreover, analysing T cells linked to grey-matter destruction to determine their role and function might improve our understanding of progressive disease and enable the development of new monitoring and treatment strategies for multiple sclerosis.

The authors' identification of $\beta$-synucleinreactive $\mathrm{T}$ cells in people who have multiple sclerosis is particularly interesting because $\mathrm{T}$ cells that recognize another protein called $\alpha$-synuclein have been found in blood samples from people with Parkinson's disease ${ }^{12}$. Both $\alpha$-synuclein and $\beta$-synuclein are present in junctions between nerve cells, called synapses, are broadly expressed throughout the nervous system, and fulfil similar functions of binding lipid membranes and regulating an intracellular transport process called endocytosis ${ }^{13}$. The emerging appreciation of the synuclein family of proteins as immune-cell targets suggests that grey-matter proteins might have a role in chronic, inflammatory neurodegenerative diseases, and that perhaps there is a connection between the development of Parkinson's disease and that of multiple sclerosis.

Future studies into synuclein-reactive $\mathrm{T}$ cells - including an analysis of how they function and change in number after immunetargeted therapies, and an investigation into whether they have a role in other neurological diseases - might unveil previously unknown mechanisms that cause multiple sclerosis or other types of neurodegeneration. Moreover, such studies might aid the development of animal models for investigating progressive multiple sclerosis.

Jenna L. Pappalardo and David A. Hafler are in the Departments of Neurology and Immunobiology, Yale School of Medicine, New Haven, Connecticut 06520, USA.

D.A.H. is also at The Broad Institute of MIT and Harvard University, Cambridge, Massachusetts.

e-mails: jenna.pappalardo@yale.edu; david.hafler@yale.edu

1. The International Multiple Sclerosis Genetics Consortium. Nature 476, 214-219 (2011).

2. Lodygin, D. et al. Nature $566,503-(2019)$.

3. Stern, J. N. et al. Sci. Transl. Med. 6 , 248ra107248ra107 (2014).

4. Dendrou, C. A., Fugger, L. \& Friese, M. A. Nature Rev. Immunol. 15, 545-558 (2015).

5. Ota, K. et al. Nature 346, 183-187 (1990).

6. Cao, Y. et al. Sci. Transl. Med. 7, 287 ra74 (2015).

7. Jelcic, l. et al. Cell 175, 85-100 (2018).

8. Lucchinetti, C. F. et al. N. Engl. J. Med. 365, 2188-2197 (2011).

9. Calabrese, M. et al. Brain 135, 2952-2961 (2012).

10.Brown, J. W. L. et al. J. Am. Med. Assoc. 321, 175-187 (2019).

11.Mor, F., Quintana, F., Mimran, A. \& Cohen, I. R. J. Immunol. 170, 628-634 (2003).

12.Sulzer, D. et al. Nature 546, 656-661 (2017)

13.Vargas, K. J. et al. J. Neurosci. 34, 9364-9376 (2014).

D.A.H. declares competing financial interests. See go.nature.com/2tknlg2 for details.

This article was published online on 20 February 2019. 